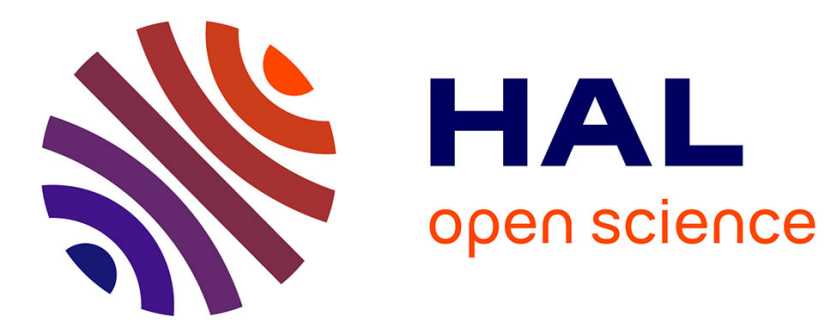

\title{
Performances of the Virgo interferometer longitudinal control system
}

F. Acernese, F. Antonucci, S. Aoudia, K.G. Arun, P. Astone, G. Ballardin, F. Barone, M. Barsuglia, Th.S. Bauer, M.G. Beker, et al.

\section{- To cite this version:}

F. Acernese, F. Antonucci, S. Aoudia, K.G. Arun, P. Astone, et al.. Performances of the Virgo interferometer longitudinal control system. Astroparticle Physics, 2009, 33 (2), pp.75. 10.1016/j.astropartphys.2009.11.006 . hal-00521252

\section{HAL Id: hal-00521252 https://hal.science/hal-00521252}

Submitted on 27 Sep 2010

HAL is a multi-disciplinary open access archive for the deposit and dissemination of scientific research documents, whether they are published or not. The documents may come from teaching and research institutions in France or abroad, or from public or private research centers.
L'archive ouverte pluridisciplinaire HAL, est destinée au dépôt et à la diffusion de documents scientifiques de niveau recherche, publiés ou non, émanant des établissements d'enseignement et de recherche français ou étrangers, des laboratoires publics ou privés. 


\section{Accepted Manuscript}

Performances of the Virgo interferometer longitudinal control system

F. Acernese, F. Antonucci, S. Aoudia, K.G. Arun, P. Astone, G. Ballardin, F. Barone, M. Barsuglia, Th.S. Bauer, M.G. Beker, S. Bigotta, S. Birindelli, M.A. Bizouard, M. Blom, C. Boccara, F. Bondu, L. Bonelli, L. Bosi, S. Braccini, C. Bradaschia, A. Brillet, V. Brisson, R. Budzyński, T. Bulik, H.J. Bulten, D. Buskulic, G. Cagnoli, E. Calloni, E. Campagna, B. Canuel, F. Carbognani, F. Cavalier, R. Cavalieri, G. Cella, E. Cesarini, E. Chassande-Mottin, A. Chincarini, F. Cleva, E. Coccia, C.N. Colacino, J. Colas, A. Colla, M.

Colombini, C. Corda, A. Corsi, J.-P. Coulon, E. Cuoco, S. D’Antonio, A. Dari, V. Dattilo, M. Davier, R. Day, R. De Rosa, M. del Prete, L. Di Fiore, A. Di Lieto, M. Di Paolo Emilio, A. DiVirgilio, A. Dietz, M. Drago, V. Fafone, I. Ferrante, F. Fidecaro, I. Fiori, R. Flaminio, J.-D. Fournier, J. Franc, S. Frasca, F. Frasconi, A. Freise, L. Gammaitoni, F. Garufi, G. Gemme, E. Genin, A. Gennai, A. Giazotto, M. Granata, C. Greverie, G. Guidi, H. Heitmann, P. Hello, S. Hild, D. Huet, P. Jaranowski, I. Kowalska, A. Królak, P. La Penna, N. Leroy, N. Letendre, T.G.F. Li, M. Lorenzini, V. Loriette, G. Losurdo, J.-M.

Mackowski, E. Majorana, N. Man, M. Mantovani, F. Marchesoni, F. Marion, J. Marque, F. Martelli, A. Masserot, F. Menzinger, C. Michel, L. Milano, Y. Minenkov, M. Mohan, J. Moreau, N. Morgado, A. Morgia, S. Mosca, V. Moscatelli, B. Mours, I. Neri, F. Nocera, G. Pagliaroli, C. Palomba, F. Paoletti, S. Pardi, M. Parisi, A. Pasqualetti, R. Passaquieti, D. Passuello, G. Persichetti, M. Pichot, F. Piergiovanni, M. Pietka, L. Pinard, R. Poggiani, M. Prato, G.A. Prodi, M. Punturo, P. Puppo, O. Rabaste, D.S. Rabeling, P. Rapagnani, V. Re, T. Regimbau, F. Ricci, F. Robinet, A. Rocchi, L. Rolland, R. Romano, D. Rosińska, P. Ruggi, F. Salemi, B. Sassolas, D. Sentenac, R. Sturani, B. Swinkels, A. Toncelli, M. Tonelli, E. Tournefier, F. Travasso, J. Trummer, G. Vajente, J.F.J. van den Brand, S. van der Putten, M. Vavoulidis, G. Vedovato, D. Verkindt, F. Vetrano, A. Viceré, J.-Y. Vinet, H. Vocca, M. Was, M. Yvert

PII:

DOI:

Reference:

To appear in:

Received Date:

Revised Date:

Accepted Date:
S0927-6505(09)00176-5

10.1016/j.astropartphys.2009.11.006

\section{ASTPHY 1450}

\section{Astroparticle Physics}

30 September 2009

10 November 2009

11 November 2009

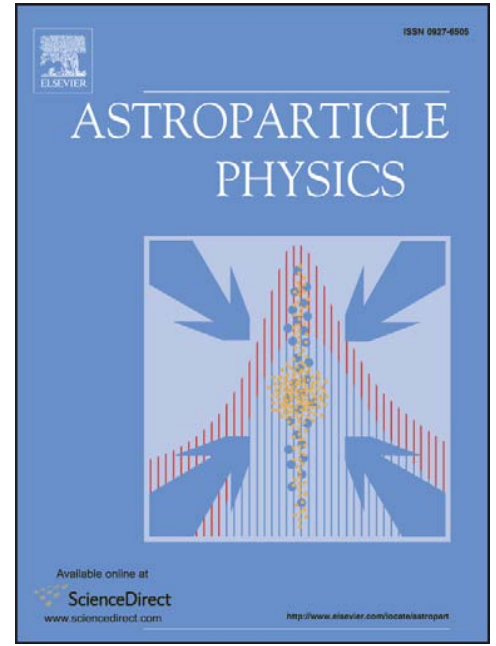


Please cite this article as: F. Acernese, F. Antonucci, S. Aoudia, K.G. Arun, P. Astone, G. Ballardin, F. Barone, M. Barsuglia, Th.S. Bauer, M.G. Beker, S. Bigotta, S. Birindelli, M.A. Bizouard, M. Blom, C. Boccara, F. Bondu, L. Bonelli, L. Bosi, S. Braccini, C. Bradaschia, A. Brillet, V. Brisson, R. Budzyński, T. Bulik, H.J. Bulten, D. Buskulic, G. Cagnoli, E. Calloni, E. Campagna, B. Canuel, F. Carbognani, F. Cavalier, R. Cavalieri, G. Cella, E. Cesarini, E. Chassande-Mottin, A. Chincarini, F. Cleva, E. Coccia, C.N. Colacino, J. Colas, A. Colla, M. Colombini, C. Corda, A. Corsi, J.-P. Coulon, E. Cuoco, S. D’Antonio, A. Dari, V. Dattilo, M. Davier, R. Day, R. De Rosa, M. del Prete, L. Di Fiore, A. Di Lieto, M. Di Paolo Emilio, A. DiVirgilio, A. Dietz, M. Drago, V. Fafone, I. Ferrante, F. Fidecaro, I. Fiori, R. Flaminio, J.-D. Fournier, J. Franc, S. Frasca, F. Frasconi, A. Freise, L. Gammaitoni, F. Garufi, G. Gemme, E. Genin, A. Gennai, A. Giazotto, M. Granata, C. Greverie, G. Guidi, H. Heitmann, P. Hello, S. Hild, D. Huet, P. Jaranowski, I. Kowalska, A. Królak, P. La Penna, N. Leroy, N. Letendre, T.G.F. Li, M. Lorenzini, V. Loriette, G. Losurdo, J.-M. Mackowski, E. Majorana, N. Man, M. Mantovani, F. Marchesoni, F. Marion, J. Marque, F. Martelli, A. Masserot, F. Menzinger, C. Michel, L. Milano, Y. Minenkov, M. Mohan, J. Moreau, N. Morgado, A. Morgia, S. Mosca, V. Moscatelli, B. Mours, I. Neri, F. Nocera, G. Pagliaroli, C. Palomba, F. Paoletti, S. Pardi, M. Parisi, A. Pasqualetti, R. Passaquieti, D. Passuello, G. Persichetti, M. Pichot, F. Piergiovanni, M.Pietka, L. Pinard, R. Poggiani, M. Prato, G.A. Prodi, M. Punturo, P. Puppo, O. Rabaste, D.S. Rabeling, P. Rapagnani, V. Re, T. Regimbau, F. Ricci, F. Robinet, A. Rocchi, L. Rolland, R. Romano, D. Rosińska, P. Ruggi, F. Salemi, B. Sassolas, D. Sentenac, R. Sturani, B. Swinkels, A. Toncelli, M. Tonelli, E. Tournefier, F. Travasso, J. Trummer, G. Vajente, J.F.J. van den Brand, S. van der Putten, M. Vavoulidis, G. Vedovato, D. Verkindt, F. Vetrano, A. Viceré, J.-Y. Vinet, H. Vocca, M. Was, M. Yvert, Performances of the Virgo interferometer longitudinal control system, Astroparticle Physics (2009), doi: 10.1016/j.astropartphys.2009.11.006

This is a PDF file of an unedited manuscript that has been accepted for publication. As a service to our customers we are providing this early version of the manuscript. The manuscript will undergo copyediting, typesetting, and review of the resulting proof before it is published in its final form. Please note that during the production process errors may be discovered which could affect the content, and all legal disclaimers that apply to the journal pertain. 


\section{Performances of the Virgo interferometer longitudinal control system}

F. Acernese ${ }^{6 a c}$, F. Antonucci ${ }^{9 a}$, S. Aoudia ${ }^{15 a}$, K.G. Arun ${ }^{11}$, P. Astone ${ }^{9 a}$, G. Ballardin $^{2}$, F. Barone ${ }^{6 a c}$, M. Barsuglia ${ }^{1}$, Th.S. Bauer ${ }^{14 a}$, M.G. Beker ${ }^{14 a}$, S. Bigotta $^{8 a b}$, S. Birindelli ${ }^{15 a}$, M.A. Bizouard ${ }^{11}$, M. Blom ${ }^{14 a}$, C. Boccara ${ }^{3}$, F. Bondu $^{15 b}$, L. Bonelli ${ }^{8 a b}$, L. Bosi ${ }^{7 a}$, S. Braccini ${ }^{8 a}$, C. Bradaschia ${ }^{8 a}$, A.

Brillet $^{15 a}$, V. Brisson ${ }^{11}$, R. Budzyński1 ${ }^{17 b}$, T. Bulik ${ }^{17 c d}$, H.J. Bulten ${ }^{14 a b}$, D. Buskulic $^{12}$, G. Cagnoli ${ }^{4 a}$, E. Calloni ${ }^{6 a b}$, E. Campagna ${ }^{4 a c}$, B. Canuel ${ }^{2}$, F. Carbognani $^{2}$, F. Cavalier ${ }^{11}$, R. Cavalieri ${ }^{2}$, G. Cella ${ }^{8 a}$, E. Cesarini ${ }^{4 c}$, E.

Chassande-Mottin ${ }^{1}$, A. Chincarini ${ }^{5}$, F. Cleva ${ }^{15 a}$, E. Coccia ${ }^{10 a b}$,

C. N. Colacino*, ${ }^{* *}$, J. Colas ${ }^{2}$, A. Colla ${ }^{9 a b}$, M. Colombini ${ }^{9 b}$, C. Corda ${ }^{8 a b}$, A. Corsi $^{9 a}$, J.-P. Coulon ${ }^{15 a}$, E. Cuoco ${ }^{2}$, S. D'Antonio ${ }^{10 a}$, A. Dari ${ }^{7 a b}$, V. Dattilo ${ }^{2}$,

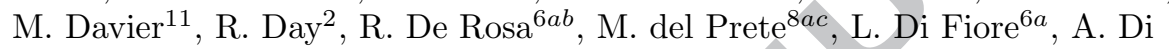

Lieto $^{8 a b}$, M. Di Paolo Emilio ${ }^{10 a d}$, A. Di Virgilio ${ }^{8 a}$, A. Dietz ${ }^{*}, 12$, M. Drago ${ }^{16 c d}$,

V. Fafone ${ }^{10 a b}$, I. Ferrante ${ }^{8 a b}$, F. Fidecaro ${ }^{8 a b}$, I. Fiori ${ }^{2}$, R. Flaminio ${ }^{13}$, J.-D.

Fournier $^{15 a}$, J. Franc ${ }^{13}$, S. Frasca ${ }^{9 a b}$, F. Frasconi ${ }^{8 a}$, A. Freise ${ }^{* * *}$, L.

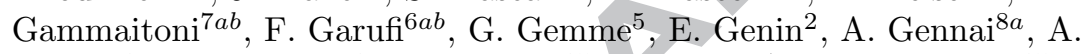

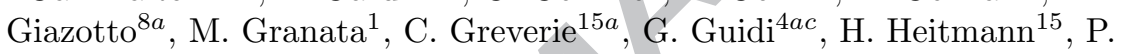
Hello $^{11}$, S. Hild ${ }^{* * *}$, D. Huet ${ }^{2}$, P. Jaranowski ${ }^{17 e}$, I. Kowalska ${ }^{17 c}$, A. Królak ${ }^{17 a f}$, P. La Penna ${ }^{2}$, N. Leroy ${ }^{11}$, N. Letendre ${ }^{12}$, T.G.F. Li $^{14 a}$, M. Lorenzini ${ }^{4 a}$, V. Loriette $^{3}$, G. Losurdo ${ }^{4 a}$, J.-M. Mackowski ${ }^{13}$, E. Majorana ${ }^{9 a}$, N. Man ${ }^{15 a}$, M.

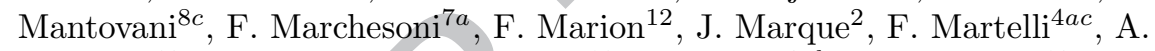
Masserot $^{12}$, F. Menzinger ${ }^{2}$, C. Michel ${ }^{13}$, L. Milano ${ }^{6 a b}$, Y. Minenkov ${ }^{10 a}$, M.

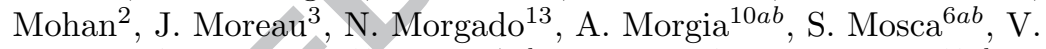
Moscatelli $^{9 a}$, B. Mours ${ }^{12}$, I. Neri ${ }^{7 a b}$, F. Nocera ${ }^{2}$, G. Pagliaroli ${ }^{10 a b}$, C. Palomba $^{9 a}$, F. Paoletti ${ }^{8 a, 2}$, S. Pardi ${ }^{6 a b}$, M. Parisi ${ }^{6 b}$, A. Pasqualetti ${ }^{2}$, R. Passaquieti $^{8 a b}$, D. Passuello ${ }^{8 a}$, G. Persichetti ${ }^{6 a b}$, M. Pichot ${ }^{15 a}$, F.

Piergiovanni ${ }^{4 a c}$, M. Pietka ${ }^{17 e}$, L. Pinard ${ }^{13}$, R. Poggiani ${ }^{8 a b}$, M. Prato ${ }^{5}$, G.A. Prodi $^{16 a b}$, M. Punturo ${ }^{7 a}$, P. Puppo ${ }^{9 a}$, O. Rabaste ${ }^{1}$, D.S. Rabeling ${ }^{14 a b}$, P.

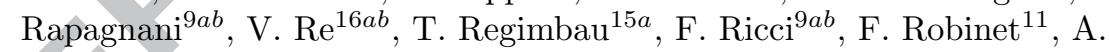

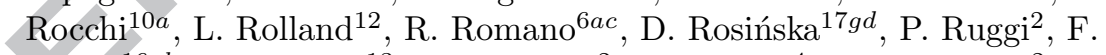
Salemi $^{16 a b}$, B. Sassolas ${ }^{13}$, D. Sentenac ${ }^{2}$, R. Sturani ${ }^{4 a c}$, B. Swinkels ${ }^{2}$, A.

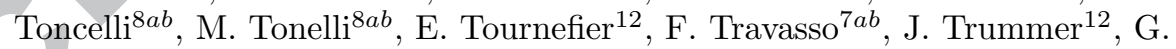
Vajente $^{8 a b}$, J.F.J. van den Brand ${ }^{14 a b}$, S. van der Putten ${ }^{14 a}$, M. Vavoulidis ${ }^{11}$, G. Vedovato ${ }^{16 c}$, D. Verkindt ${ }^{12}$, F. Vetrano ${ }^{4 a c}$, A. Viceré ${ }^{4 a c}$, J.-Y. Vinet ${ }^{15 a}$, H. Vocca $^{7 a}$, M. Was ${ }^{11}$, M. Yvert ${ }^{12}$

${ }^{1}$ AstroParticule et Cosmologie (APC), CNRS: UMR7164-IN2P3-Observatoire de Paris-Université Denis Diderot-Paris VII - CEA : DSM/IRFU

${ }^{2}$ European Gravitational Observatory (EGO), I-56021 Cascina (Pi), Italy

${ }^{3}$ ESPCI, CNRS, F-75005 Paris, France

\footnotetext{
${ }^{1}$ Corresponding author: gabriele.vajente@sns.it
} 
${ }^{4}$ INFN, Sezione di Firenze, I-50019 Sesto Fiorentino ${ }^{a}$; Università degli Studi di Firenze, I-50121 ${ }^{b}$, Firenze; Università degli Studi di Urbino 'Carlo Bo', I-61029 Urbinoc, Italy

${ }^{5}$ INFN, Sezione di Genova; I-16146 Genova, Italy

${ }^{6}$ INFN, sezione di Napoli ${ }^{a}$; Università di Napoli 'Federico II ${ }^{b}$ Complesso Universitario di Monte S.Angelo, I-80126 Napoli; Università di Salerno, Fisciano, I-84084 Salerno ${ }^{c}$, Italy

${ }^{7}$ INFN, Sezione di Perugia ${ }^{a}$; Università di Perugia ${ }^{b}$, I-6123 Perugia,Italy

${ }^{8}$ INFN, Sezione di Pisa ${ }^{a}$; Università di Pisa ${ }^{b}$ I-56127 Pisa; Università di Siena, I-53100 Siena ${ }^{c}$, Italy

${ }^{9}$ INFN, Sezione di Roma ${ }^{a}$; Università 'La Sapienza ${ }^{\text {, }}$, I-00185 Roma, Italy

${ }^{10}$ INFN, Sezione di Roma Tor Vergata $^{a}$; Università di Roma Tor Vergata ${ }^{b}$, Istituto di

Fisica dello Spazio Interplanetario (IFSI) INAFc, I-00133 Roma; Università dell'Aquila, I-67100 L'Aquila' ${ }^{\text {, Italy }}$

${ }^{11}$ LAL, Université Paris-Sud, IN2P3/CNRS, F-91898 Orsay, France

${ }^{12}$ Laboratoire d'Annecy-le-Vieux de Physique des Particules (LAPP), IN2P3/CNRS, Université de Savoie, F-74941 Annecy-le-Vieux, France

${ }^{13}$ Laboratoire des Matériaux Avancés (LMA), IN2P3/CNRS, F-69622 Villeurbanne, Lyon, France

${ }^{14}$ National institute for Subatomic Physics, P.O. Box 41882, 1009 DB ${ }^{a}$; VU University Amsterdam, De Boelelaan 1081, $1081 \mathrm{HV}^{b}$, Amsterdam, The Netherlands

${ }^{15}$ Université Nice-Sophia-Antipolis, CNRS, Observatoire de la Côte d'Azur, F-06304 Nice

${ }^{a}$; Institut de Physique de Rennes, CNRS, Université de Rennes 1, 35042 Rennes ${ }^{b}$; France

${ }^{16}$ INFN, Gruppo Collegato di Trento $^{a}$ and Università di Trento ${ }^{b}$, I-38050 Povo, Trento,

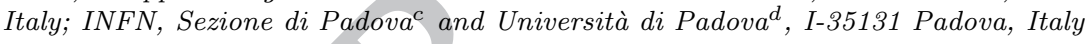

${ }^{17}$ IM-PAN 00-956 Warsaw ${ }^{a}$; Warsaw Univ. 00-681 ; Astro. Obs. Warsaw Univ. 00-478 ; CAMK-PAM 00-716 Warsaw ${ }^{d}$; Bialystok Univ. 15-424 ; IPJ 05-400 Swierk-Otwock ${ }^{f}$ Inst. of Astronomy 65-265 Zielona Gora ${ }^{g}$, Poland

*Cardiff University, Cardiff, CF24 3AA, United Kingdom

** Eötvös University, ELTE 1053 Budapest, Hungary

* University of Birmingham, Birmingham, B15 2TT, United Kingdom

\section{Abstract}

The performances of the longitudinal sensing and control system of the Virgo gravitational wave detector are described. This system is able to stably maintain the RMS residual fluctuation of the interferometer longitudinal degrees of freedom around or below $10^{-11} \mathrm{~m}$, compatible with the original Virgo requirements. Moreover the detector sensitivity is not limited by longitudinal control noise at any frequency. Indeed the noise re-introduced by the longitudinal control system does not affect the Virgo design sensitivity. 


\section{Introduction}

The Virgo gravitational wave detector [1], located at the EGO site near Pisa in Italy, is a power-recycled Michelson interferometer with 3-km long FabryPerot resonant cavities in the two arms. Any gravitational signal will be detected as a differential change of optical length of the arms proportional to the signal amplitude $h=\delta L / L$. All mirrors are suspended to high-performance multistage passive isolation systems (the super-attenuators [2]) designed to reduce the contribution of ground micro-seismic motion to mirror displacement. It ensures a good sensitivity to gravitational waves down to $10 \mathrm{~Hz}$. The input laser beam is provided by a $20 \mathrm{~W}$ Nd:Yag laser with a wavelength of $1.064 \mu \mathrm{m}$. Before entering the main part of the interferometer, it passes through an input mode cleaner (IMC) to filter its transverse mode and reduce jitter. A scheme of the Virgo detector is shown in fig. 1.

The first Virgo Science Run (VSR1) took place starting on May 18th 2007 and ending in October 1st 2007. During this period the interferometer ran in a controlled and stable configuration with the best possible sensitivity achieved in that period. Scientific data was collected in coincidence with the three LSC detectors [3].

After the end of the run, commissioning activities restarted and continued until June 2008, resulting in a large improvement of the detector sensitivity. Starting from June 2008 a programmed shutdown started to begin the implementation of minor detector improvements to move towards the Virgo+ configuration. The detector is expected to be back in operation for a second science run in the middle of 2009 .

\section{Longitudinal control}

The task of bringing the interferometer from an uncontrolled configuration to the final operating state is usually called lock acquisition. Indeed the response of the system is linear with respect to the mirror motion only in a limited range (about $10 \mathrm{~nm}$ ) around the operating point, which is defined by the resonance conditions inside the interferometer cavities. In Virgo the lock acquisition is based on the variable finesse technique which has been already described in [4]. This paper deals with the characterization and performances of the longitudinal and control system when the detector runs in steady state configuration.

The distances between all suspended mirrors must be kept fixed with subnanometer accuracy, maintaining the correct resonance conditions of the laser field inside the interferometer cavities to ensure the maximum enhancement in the detector sensitivity. The free motion of the mirrors, under local control systems, is of the order of $1 \mu \mathrm{m}$. Hence the necessity of a global longitudinal sensing and control system, capable of extracting length information from the laser fields.

Corrections are applied to mirror by means of coil-magnets actuators: magnets are attached to the rear face of each mirror and coils to a recoil mass, both suspended to the same mass at the bottom of the super-attenuator chain 


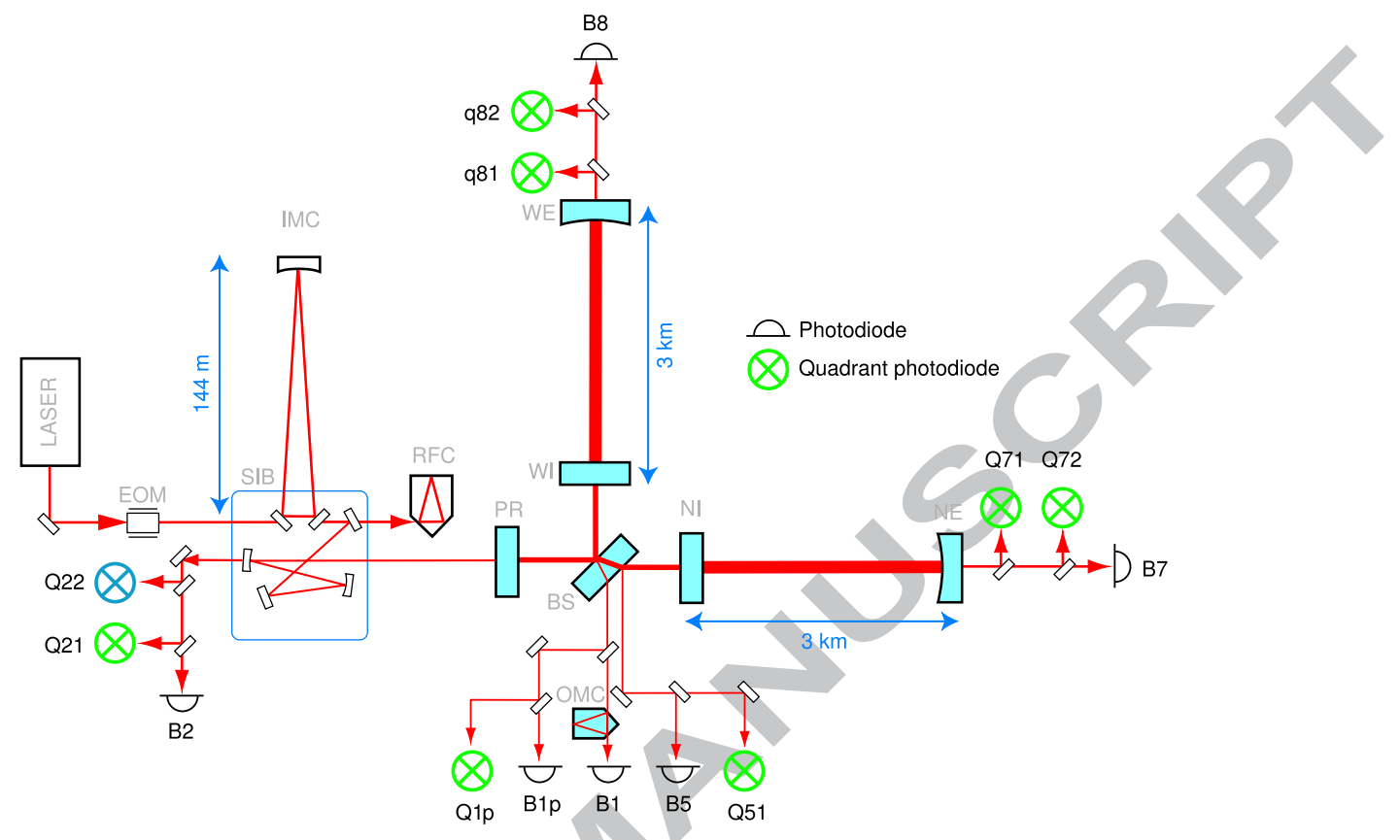

Figure 1: Optical scheme of the Virgo interferometer. The main output beams are: B1 is the main anti-symmetric port, B2 is the interferometer reflection, B5 is the pick-off beam from the secondary face of the beam splitter, B7 and B8 are the transmission of the two arm Fabry-Perot cavities.

(the marionette). In this way a force can be applied to the mirror without reintroducing seismic noise through coils attached to ground. A similar control is implemented also at the level of the marionette and of the very top superattenuator stage. In this way a hierarchical control of the mirror longitudinal motion is possible.

The technique used in Virgo, as well in all other similar experiments, is based on frontal modulation [5], an extension of the Pound-Drever-Hall technique [6]. The main laser beam is phase modulated, before being injected inside the main interferometer, at $6 \mathrm{MHz}$ and $8 \mathrm{MHz}$. The carrier and resulting radio-frequency sideband fields behave differently inside the interferometer: at the operating point the carrier is resonant inside the arm cavities, resonant inside the powerrecycling cavity and undergoes destructive interference at the anti-symmetric port (dark fringe condition). The $6 \mathrm{MHz}$ sidebands are resonant in the power recycling cavity but not in the arm cavities. The difference between the two short Michelson arms (the distances between the beam splitter and the two input mirrors) is called Schnupp asymmetry. It is about $0.8 \mathrm{~m}$ to give a significant transmission of $6 \mathrm{MHz}$ sidebands to the anti-symmetric port. Finally the $8 \mathrm{MHz}$ sidebands are resonant nowhere and therefore they are reflected by the entire interferometer. 
Many photo-detectors are placed on all the main output beams, see fig. 1. The output of each photo-detector is demodulated at $6 \mathrm{MHz}$ to extract signals proportional to the various longitudinal degrees of freedom motions. The only photo-detector demodulated at $8 \mathrm{MHz}$ is the one placed on the interferometer reflection.

The optical distances between the mirrors are usually expressed in terms of the physical degrees of freedom to be controlled [7]: the common and differential relative change of the two arm cavity lengths (CARM and DARM respectively); the differential motion of the short Michelson interferometer arms (MICH); the power recycling cavity length (PRCL):

$$
\begin{aligned}
D A R M & =L_{N I-N E}-L_{W I-W E} \\
C A R M & =\frac{L_{N I-N E}+L_{W I-W E}}{2} \\
M I C H & =L_{B S-N I}-L_{B S-W I} \\
P R C L & =L_{P R-B S}+\frac{L_{B S-N I}-L_{B S-W I}}{2}
\end{aligned}
$$

Any CARM motion is equivalent to a laser frequency shift, therefore the control is implemented in such a way that the laser frequency follows the fluctuation in the mean cavities length (second stage of frequency stabilization SSFS [8]), as sensed by the in-phase $6 \mathrm{MHz}$ demodulation of the beam splitter pick-off beam B5. To prevent the cavities to move freely in their common mode, an additional control is implemented: the common mode of the two arms is controlled by maintaining the laser frequency resonant inside a rigid reference cavity (RFC) placed on a pick-off beam in transmission of the input mode cleaner. This is the mechanical CARM control to which this paper refers later on.

The other auxiliary degrees of freedom are controlled with feedback systems using error signals coming from different ports: PRCL is controlled with the quadrature demodulated signal from $\mathrm{B} 5$; $\mathrm{MICH}$ using the $8 \mathrm{MHz}$ in-phase demodulation of the interferometer reflected beam B2; DARM using the in-phase signal of the dark fringe B1 demodulated at $6 \mathrm{MHz}$. The latter is clearly also the main gravitational wave channel.

To maintain the system inside the linear response range, the residual fluctuation of all degrees of freedom must be maintained below maximum total RMS values, which are listed in tab. 1 . These affect mainly the very low frequency bands, below $5 \mathrm{~Hz}$, where the main real residual mirror motions are concentrated. More stringent requirements inside the detection band (frequencies above $10 \mathrm{~Hz}$ ) come from the need that the noise re-introduced there must be lower than the detector sensitivity.

To further reduce the coupling of auxiliary degree of freedom control systems to the main gravitational channel, noise subtraction techniques are used, in a feed-forward-like manner. They are explained in more details later on in this paper. 


\begin{tabular}{cccc} 
D.O.F. & Requirements $[\mathrm{m}]$ & \multicolumn{2}{c}{ Accuracy $[\mathbf{m}]$} \\
& & VSR1 & May 2008 \\
\hline DARM & $3 \cdot 10^{-11}$ & $3 \cdot 10^{-12}$ & $1 \cdot 10^{-15}$ \\
PRCL & $2 \cdot 10^{-10}$ & $3 \cdot 10^{-11}$ & $1 \cdot 10^{-12}$ \\
MICH & $5 \cdot 10^{-10}$ & $8 \cdot 10^{-11}$ & $3 \cdot 10^{-11}$ \\
CARM & & $4 \cdot 10^{-8}$ & $2 \cdot 10^{-8}$ \\
\hline
\end{tabular}

Table 1: Requirements and obtained performances of the longitudinal control loops in terms of residual total RMS motion of the main degrees of freedom.

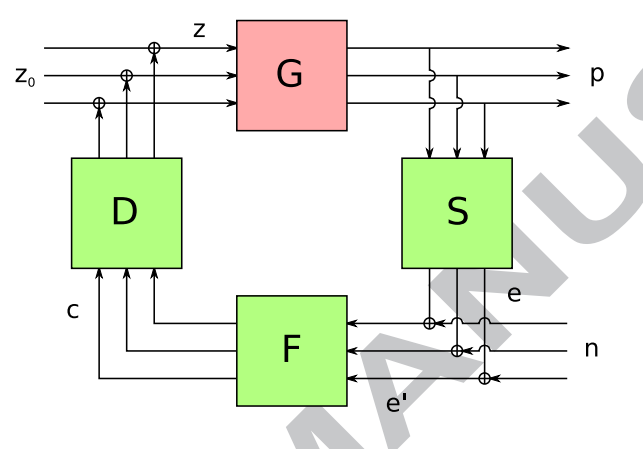

Figure 2: Scheme of the longitudinal control loops: $\mathrm{G}$ is the unknown optical matrix, $\mathrm{S}$ is the sensing matrix, $\mathrm{F}$ is the set of control filters, $\mathrm{D}$ is the driving and actuator matrix.

\section{Control system characterization}

The longitudinal control system characteristics and performances can be measured adding a suitable external perturbation to the error or control signal of the four loops $[7,10]$. Several transfer functions can be measured in this way. Assuming the noise to be added to the error point, the transfer function from the signal immediately after to immediately before the addition point gives an estimate of the open loop gain of the system, and allows characterizing the noise suppression and stability performances [13].

The most interesting measurement that can be obtained in such a way is the optical matrix of the interferometer, namely the set of all transfer functions from degree of freedom motions to photo-diode outputs. Clearly the measurement must be performed in closed-loop configuration, that is with all the longitudinal loop active and the interferometer in its low noise operating configuration. The effect of the control system must be correctly modeled and factorized out from the raw measurements.

Referring to fig. 2, the longitudinal control loops can be divided in four main parts:

- the unknown response of the interferometer (from one d.o.f. motion to one photo-diode output) is given by the optical matrix $G$; 


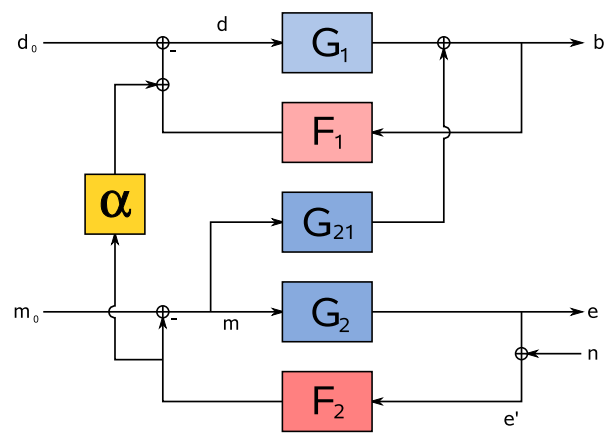

Figure 3: Scheme of the longitudinal loops involved in the noise subtraction technique. The effect of the DARM and auxiliary loops are modeled with their optical transfer functions $F_{1}$ and $F_{M}$ and with the corresponding corrector filters $G_{1}$ and $G_{M}$.

- the photo-diode signals are mixed together and normalized with a known sensing matrix $S$;

- the resulting error signal for each d.o.f. is passed through a separate control filter: the filtering matrix $F$ is therefore a known diagonal one;

- finally the correction signals for all degrees of freedom are sent in known ratios to various mirror actuators: the driving matrix contains these splitting coefficients as well as the actuator response functions.

To measure the optical matrix, an external perturbation $n$ was added to each error signal separately and the set of transfer functions $A$ from $n$ to the signal immediately after the injection point was measured. This transfer function is given by the simple equation:

$$
A=(1-S G D F)^{-1}
$$

which can be inverted to yield the optical matrix as a function of the measurement and of all the known control system parameters:

$$
G=S^{-1}\left(1-A^{-1}\right) F^{-1} D^{-1}
$$

This kind of measurement has been repeated routinely during all the science run and allowed a precise reconstruction of the interferometer optical matrix between about 10 and few hundreds Hz. The resulting optical matrix showed small frequency dependences apart from the Fabry-Perot cavity pole at about $500 \mathrm{~Hz}$. Therefore the optical matrix can be summarized as a set of simple numbers:

$$
\left(\begin{array}{c}
e_{D A R M} \\
e_{P R C L} \\
e_{M I C H} \\
e_{C A R M}
\end{array}\right)=\left[\begin{array}{cccc}
770 & 31 & 0.90 & 8.1 \\
0 & 0.068 & 0.033 & 0 \\
0 & 0.021 & 0.71 & 0 \\
0 & 0 & 0 & 5.0
\end{array}\right]\left(\begin{array}{c}
z_{D A R M} \\
z_{P R C L} \\
z_{M I C H} \\
z_{C A R M}
\end{array}\right)
$$


where $e$ and $z$ denote respectively the photo-diode error signal and the motion of each degree of freedom. All numbers are in $\mathrm{W} / \mu \mathrm{m}$, except those in the last row which are in $\mathrm{V} / \mu \mathrm{m}$. Some of the matrix elements are zero, meaning that the coupling was so small that it was not detectable given the maximum possible external perturbation. The typical uncertainties on the optical matrix elements is smaller than $10 \%$.

These results shows a good decoupling of the DARM error signal from the other auxiliary degrees of freedom. On the contrary the MICH and PRCL ones are largely coupled. This possible problem is solved by enforcing a gain hierarchy of the two loops: the PRCL control loop has a significantly higher gain than the MICH one: therefore inside the MICH loop active band-width the PRCL residual motion can be neglected and the two signals are automatically decoupled by the control system.

From these measurements it was possible to estimate the locking accuracy, that is the total RMS of the residual degree of freedom motions, see tab. 1. Already during the science run the accuracy met the requirements with large safety margins. The commissioning activities carried out after the end of VSR1 allowed to obtain much better accuracies. This was the result of many improvements: the reduction of error signal noise; the implementation of control filters with higher low frequency gain; the improvements of the low frequency suspension control. The latest performances of the longitudinal control system in terms of accuracy is also shown in tab. 1.

\section{Noise couplings to gravitational channel}

The most stringent requirements on the longitudinal sensing and control system come from the requirement that the residual motion of the auxiliary degrees of freedom does not contribute to limit the detector sensitivity. In other words the longitudinal control noise must be kept below the measured noise curve.

The contribution of each longitudinal control loop to the gravitational wave channel noise can be measured using the linear noise projection technique [9]. In brief, an external perturbation is again added inside one of the loops (to the error signal in the Virgo configuration) and the transfer function from after the perturbation point to the gravitational channel is measured. This transfer function can be used, outside the active band-width of the loop, to project the error signal noise in normal state to the main channel. This procedure is completely automated in Virgo and it has been performed routinely during the entire science run [12]. Typical results are shown in fig. 4.

The coupling of auxiliary loop noises turned out to be very large and indeed it would be the dominant source of gravitational channel noise below 300-400 Hz. To overcome this problem, noise subtraction techniques have been very efficiently implemented in Virgo for all three auxiliary degree of freedom [11]. In general, referring to fig. 3 , the residual motion of one auxiliary degree of freedom couples directly to the gravitational channel with an unknown transfer function $G_{21}$. An additional frequency dependent coupling $\alpha$ is added inside the control 
system, at the level of the driving part. The final goal is to tune $\alpha$ as well as possible to cancel the intrinsic noise coupling. This is indeed possible since the main dark fringe signal can be written as

$$
b=-\frac{1}{1+G_{1} F_{1}}\left(\alpha G_{1}+G_{21}\right) F_{2} e^{\prime}
$$

where $e^{\prime}$ is the total noise in the auxiliary loop error signal. Adding an external perturbation $N$ to this point, it is possible to measure with very good accuracy the transfer function to the gravitational channel and to compute the optimal value of the noise subtraction coefficient. The tuning is performed in an iterative manner: the first measurement is done with $\alpha=0$. An estimate of a new alpha is obtained simply inverting eq. 3. The measurement is repeated with the new $\alpha$ in operation and a refinement is found again inverting eq. 3. This iterative scheme converges quite rapidly to a good estimate of the noise subtraction coefficient: going from scratch to the final results takes normally no more than 3 iterations.

The analysis of the long term stability of the noise subtraction performances showed that the frequency dependence of $\alpha$ is constant with an accuracy better than $1 / 1000$, while the global gain can change up to $10 \%$, with typical slow variations on a time scale of hours. This is understood considering that the shape is determined by differences in the electro-mechanical response of the DARM and $\mathrm{MICH}$ actuators, while the gain is mainly driven by optical parameters of the detector, such as the arm cavity finesses, which can change with slow trends. To cope with this effect and to maintain a performant noise subtraction over long periods, a slow servo system has been implemented to continuously adjust the subtraction gain. It uses as error signal the demodulation of a calibration line added on purpose to the auxiliary degree of freedom corrections. It corrects for the noise subtraction gain with a time scale of minutes. This band-width proved to be sufficient in all standard conditions, since no faster variations of the noise coupling has ever been observed.

This technique has been implemented before the science run for the $\mathrm{MICH}$ and PRCL degrees of freedom, and afterward for the CARM one. After the end of VSR1, the MICH noise subtraction was able to reduce the normal noise coupling by a factor about 1000 between 10 and $200 \mathrm{~Hz}$, putting the corresponding control noise well below the measured sensitivity and at a level compatible with the design one. Similar noise subtractions have been implemented for PRCL and CARM, with less efficiency: PRCL noise is reduced by more than a factor 10 between 50 and $300 \mathrm{~Hz}$ and CARM noise by a factor 100 between 3 and $80 \mathrm{~Hz}$. These performances were all sufficient to avoid longitudinal control noise to limit the detector sensitivity at any frequency.

\section{Conclusions}

The longitudinal sensing and control system of the Virgo detector proved to be very stable and robust during all the duration of the first Virgo science run, allowing maintaining a continuous operation of the interferometer for as long as 90 hours and total duty cycle of $84 \%$. 

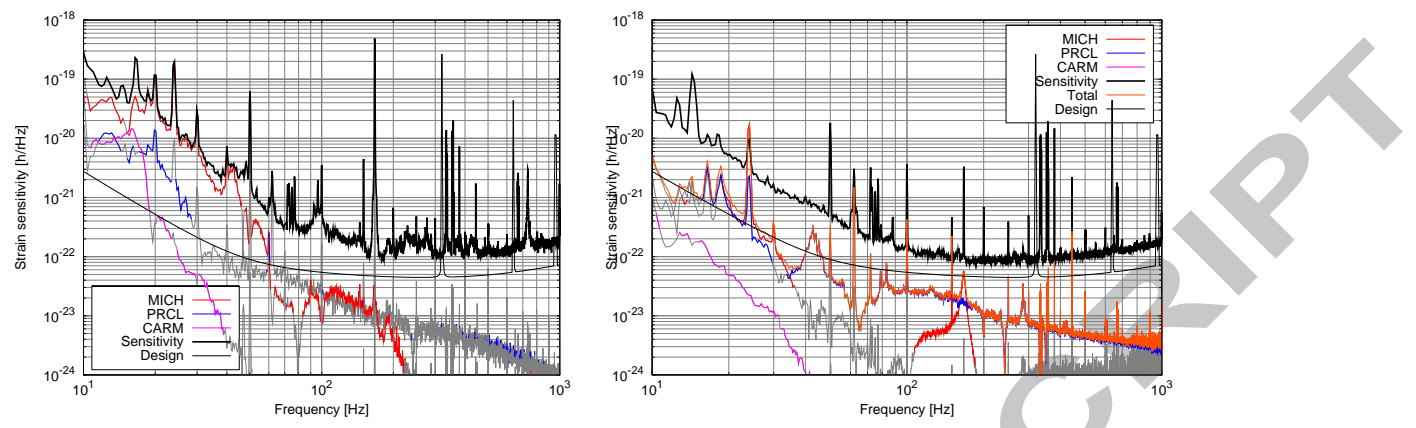

Figure 4: Measured contribution of auxiliary loop control noises to the detector sensitivity. On the left the typical performances obtained during VSR1, on the right the typical one on April 2008. The curves are grey at those frequencies where the noise coupling was so low that it was not possible to measure it.

The performances have been analyzed both in terms of locking accuracy and noise re-introduction. The measured accuracy was well below the original requirements: after the post-run commissioning improvements the three main longitudinal degrees of freedom (DARM, MICH and PRCL) showed a residual RMS motion lower than $5 \cdot 10^{-11} \mu \mathrm{m}$, with a DARM accuracy of $10^{-15} \mu \mathrm{m}$.

Finally, the contribution of longitudinal control noise has been largely reduced in the post-run commissioning period, allowing the Virgo detector to run without being limited by it at any frequency. This has been possible mainly through the optimization of noise subtraction techniques, which allowed a reduction of a factor 1000 of noise coupling during normal operations.

\section{Acknowledgments}

The authors gratefully acknowledge the support of the Italian Istituto Nazionale di Fisica Nucleare and the French Centre National de la Recherche Scientique for the construction and operation of the Virgo detector.

The authors also gratefully acknowledge the support of the research by the Foundation for Fundamental Research on Matter of the Netherlands Organisation for Scientific Research, the Polish Ministry of Science and Higher Education grant N N203 387237, the FOCUS Programme of Foundation for Polish Science and the European Associated Laboratory Astrophysics Poland-France.

\section{References}

[1] F. Acernese et al. [Virgo collaboration], Status of Virgo in Proceeding of the 7th Amaldi Conference, published in Class. Quant. Grav. 25 No. 11 (2008) 114045 
[2] S. Braccini et al., "Measurement of the seismic attenuation performance of the VIRGO super-attenuator", Astropart. Phys. 23 (2005) 557.

[3] D. Sigg [LIGO Scientific Collaboration], "Status Of The Ligo Detectors", Class. Quant. Grav. 25 (2008) 114041.

[4] F. Acernese et al. [Virgo collaboration], Lock acquisition of the Virgo gravitational wave detector, Astropart. Phys. (2008) [http://dx.doi.org/10.1016/j.astropartphys.2008.06.005] (2008)

[5] P. S. Saulson, "Fundamentals of Interferometric Gravitational Wave Detectors", World Scientific Publishing (1994)

[6] R. W. Drever, J. L. Hall, F. V. Kowalski, J. Hough, G. M. Ford, A. J. Munley and $\mathrm{H}$. Ward, "Laser phase and frequency stabilization using an optical resonator", Appl. Phys. B 97 (1983) 31

[7] G. Vajente, Analysis of sensitivity and noise sources for the Virgo gravitational wave interferometer, $\mathrm{Ph}$. D. thesis, Scuola Normale Superiore di Pisa (2008)

[8] F. Acernese et al. [Virgo collaboration], A laser with and in-loop frequency stability of $1.0 \times 10^{-21}$ on a $100 \mathrm{~ms}$ time-scale for gravitational wave detection, submitted to Phys. Rev. Lett. (2008)

[9] J. R. Smith, P. Ajith, H. Grote, M. Hewitson, S. Hild, H. Lück, K. A. Strain, B. Willke, J. Hough and K. Danzmann, "Linear projection of technical noise for interferometric gravitational-wave detectors", Class. Quant. Grav. 23 (2006) 527

[10] G. Vajente, E. Campagna, B.Swinkels, Locking characterization, Virgo internal note VIR-005A-08 (2008)

[11] B. Swinkels, E. Campagna, G. Vajente, L. Barsotti and M. Evans, Longitudinal noise subtraction: the alpha-, beta- and gammatechnique, Virgo internal note VIR-050A-08 (2008) [https://pub3.egogw.it/codifier/index.php?content $=$ show \&doc $=2055]$

[12] G. Vajente, Measurement of control noise budget, Virgo internal note VIR-003A-08 (2008) [https://pub3.egogw.it/codifier/index.php?content $=$ show\&doc $=1957]$

[13] J. Doyle, B. Francis and A. Tannenbaum, "Feedback control theory", Macmillan Publishing Co. (1990) 